\title{
Gamma-Ray Induced Luminescence of Silica Gel
}

\author{
I. Thermoluminescence and the Effect of Adsorbed Molecules
}

\author{
P. Kirmaier and J. G. Rabe \\ Hahn-Meitner-Institut für Kernforschung Berlin GmbH, Ber. Strahlenchemie, Berlin
}

(Z. Naturforsch. 30 a, 161-169 [1975] ; received December 3, 1974)

\begin{abstract}
The thermoluminescence glow curve of $\gamma$-irradiated silica gel investigated between $77^{\circ} \mathrm{K}$ and $600^{\circ} \mathrm{K}$ is a broad band extending from $80^{\circ} \mathrm{K}$ to $\sim 400^{\circ} \mathrm{K}$. The emission occurs at $\sim 450 \mathrm{~nm}$. Shallow traps $(0.1-0.2 \mathrm{eV})$ are responsible for most of the light emitted. The distribution of trap depth and the increase of trap density toward the band edge is tentatively attributed to the disordered structure of silica gel in accordance with previous suggestions concerning glasses. In contrast to the growth with radiation dose of color- and paramagnetic center concentration, luminescence is already saturated at about 1 Mrad. The luminescence decay follows the hyperbolic relation derived by Adirowich for a bimolecular process in the case of retrapping for which evidence is presented. At very low radiation doses the initial intensity of luminescence was proportional to the square of the dose rate as expected for a bimolecular reaction.

Removal of surface $\mathrm{OH}$ groups as accomplished by raising the degassing temperature of the gel results in an increase of luminescence intensity by several orders of magnitude. Hydrogen and oxygen when adsorbed prior to or subsequent to irradiation reduce the luminescence intensity to about one per cent of that observed in vacuum or in the presence of helium. The results are discussed in terms of surface reactions involving free and trapped charge carriers generated during irradiation.
\end{abstract}

\section{Introduction}

Marked changes in the chemical activity of high surface area solids subjected to ionizing radiation have been observed ${ }^{1,2}$. Generally, insulators (i. e. solids with a wide band gap) were found to exhibit the largest changes. Reactions of adsorbed molecules present during irradiation and added subsequently to the vacuum irradiated solid have both been studied. They are explained either by energy or charge transfer from the irradiated solid to the adsorbed molecules. Energy transfer is inferred from the dependence on the width of the band gap of the surface reaction ${ }^{3,4}$, whereas charge transfer is manifested by the production of radical anions or cations $^{5-9}$.

Such reactions are closely related to the formation of trapped charge carriers. Briefly, in the band model the electronic states are assumed to form bands, in the case of dielectrics the upper completely filled band being separated from the next empty one by a band gap of several eV. Trapping of electrons and holes occurs within the band gap at localised levels.

Of the various methods available to ingestigate trapped charge-carriers, esr has been used most frequently for studies of such irradiated high surface area solids which are of interest with respect to the reactions mentioned above ${ }^{5-9}$. Optical absorption

Reprint requests to Dr. J. G. Rabe, Hahn-Meitner-Institut für Kernforschung, D-1000 Berlin 39, Postfach 390128. measurements ${ }^{10,11}$, chemical determination of $\mathrm{F}$ centers ${ }^{12}$ and thermostimulated exoelectronic emission ${ }^{13}$ have also been applied. There appear to be no studies of thermoluminescence on such solids although they give information on the distribution of trap depths, i. e. activation energies for thermal release of charges assumed to be involved in surface reactions. Thermoluminescence results from luminescent centers interacting with charges thermally released from traps. The glow curves - the current from a photomultiplier recorded as a function of sample temperature which is increased linearily may consist of one or several glow peaks representing the release of charges from individual traps.

In the present work thermoluminescence of $\gamma$-irradiated silica gel is studied with the objective of describing the trapping of charges as effected by changes of the surface properties. Accordingly, the effects of thermal treatment before irradiation and of various gases adsorbed prior to irradiation or added subsequently are investigated. Silica gel was chosen because its surface area amounts to hundreds of square meters per gram. It is, furthermore, known to yield centers of chemical activity when exposed to ionizing radiation, and has been most frequently used for the investigation of surface reactions. The stimulation of luminescence occurring on adsorption of gaseous molecules at the irradiated solid is described in the subsequent paper (referred to as II). 


\section{Experimental Section}

"Silica gel HR extra pure acc. to Stahl" was obtained from Merck, the specific surface area is $450 \mathrm{~m}^{2} / \mathrm{g}$ as stated by the supplier. The gases used were obtained from Linde. Approximately, $0.5 \mathrm{~g}$ of gel was placed in a pyrex ampoule consisting of $10 \mathrm{~mm}$ i.d. tubing extended on one side by capillary tubing of $0.6 \mathrm{~mm}$ i.d. and fitted on the other side with a break-seal. The sample was degassed on a vacuum line by heating - usually at $500^{\circ} \mathrm{C}-$ to a residual pressure of $10^{-5}$ Torr. After sealingoff, the ampoule with the gel transferred completely to the wide end was placed in a dewar containing liquid nitrogen and irradiated in a ${ }^{60} \mathrm{Co}$ source. The dose rate varied between 1.4 and $9 \times 10^{5} \mathrm{rad} / \mathrm{h}$. The dose was determined by Fricke dosimetry making appropriate corrections for the different electron densities. Prior to measurement the capillary tubing was heated in an electrical furnace while the gel in the wide tubing was kept in liquid nitrogen. The heating annealed luminescence produced in the glass. After cooling to $77^{\circ} \mathrm{K}$ the irradiated gel was transferred into the capillar and the ampoule inserted into the measuring device. Exposure of the irradiated gel to light was avoided as much as possible.

The measuring device for thermoluminescence (described in reference ${ }^{14}$ ) basically consisted of a horizontal quartz tube extending into a vertically attached U-shaped tube. A stream of air cooled by liquid nitrogen entered the horizontal part of the quartz tubing which contained a heating coil. The air passed into the U-tube cooling the ampoule which was inserted vertically in the open end of this tube. Heating of the coil was regulated by a thermal controler (L 70/171 from Linseis) measuring the temperature at the ampoule. It provided heating rates of 0.1 to $50{ }^{\circ} \mathrm{C} / \mathrm{min}$, but 5 to $10^{\circ} \mathrm{C} / \mathrm{min}$ were found suitable for heating the gel with no temperature lag between the inner and outer portions of the gel column of $0.6 \mathrm{~mm}$ diameter. To insure good thermal insulation the quartz tubing was double walled and evacuated. The sample was viewed by an EMI photomultiplier type 9558QB. The output of the photomultiplier was amplified and recorded by a $1 \mathrm{mV}$ recorder (Hartmann and Braun) as a function of time. For measurement of the emission spectrum an interference strip filter for the region 400 to $700 \mathrm{~nm}$ (Schott) was used. The filter of $60 \mathrm{~mm}$ length was moved back and forth by means of a motor across a $1 \mathrm{~mm}$ slit fixed in front of the optical window. The light emitted from the sample passed through this slit and, depending on the position of the filter, light of certain wavelengths passed through a second $1 \mathrm{~mm}$ slit to the PM tube. The TL apparatus described could be operated at temperatures from 80 to $600{ }^{\circ} \mathrm{K}$. The glow peak position for different samples were found to be reproducible within $\pm 1^{\circ}$. The 5 to $10 \%$ deviation observed in luminescence intensity is attributed to the difference of powder packing from one sample to another.

\section{Results}

\subsection{Dose Dependence of Glow Curves}

A set of glow curves obtained from samples outgassed at about $800^{\circ} \mathrm{K}$ to $\sim 10^{-5}$ Torr and irradiated to doses ranging from $2.4 \cdot 10^{3} \mathrm{rad}$ to $1.1 \cdot 10^{7} \mathrm{rad}$ is shown in Figures 1 and 2 . The glow curves are confined to the temperature region below
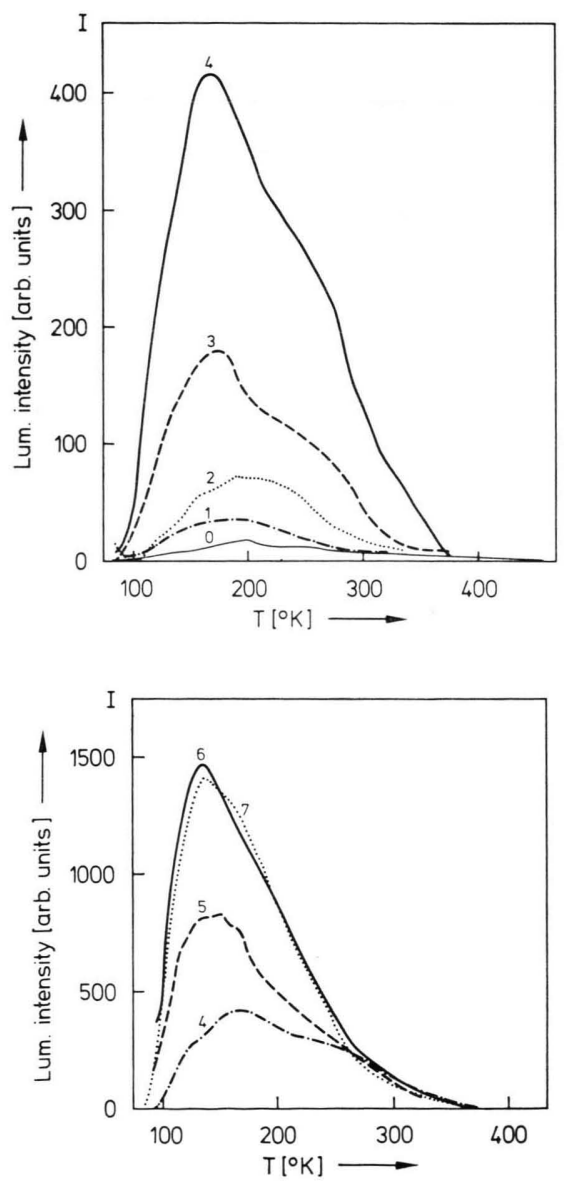

Figs. 1 and 2. TL Glow curves of silica gel outgassed at $800^{\circ} \mathrm{K}, \gamma$-irradiated at $77^{\circ} \mathrm{K}$ and heated at a rate of $5^{\circ}$ per min.

0. $2.38 \times 10^{3} \mathrm{rad} ; \quad$ 1. $1.19 \times 10^{4} \mathrm{rad} ; \quad 2.3 .38 \times 10^{4} \mathrm{rad}$; 3. $7.15 \times 10^{4} \mathrm{rad} ; \quad 4.1 .43 \times 10^{5} \mathrm{rad}$; 5. $2.88 \times 10^{5} \mathrm{rad}$; 6. $9.00 \times 10^{5} \mathrm{rad} ; \quad 7.1 .08 \times 10^{7} \mathrm{rad}$. 
$400{ }^{\circ} \mathrm{K}$ with no further peak being detectable up to $600{ }^{\circ} \mathrm{K}$, the limit of the device used. The structure of the glow curves changes as the radiation dose is increased and the rise of luminescence in intensity is accompanied by a maximum shift to lower temperatures. This may be explained by the distribution of traps in depth. Since to a first approximation more shallow traps will be emptied at lower temperatures, the maximum shift to lower temperatures would indicate that the concentration of traps increases drastically towards shallow traps. At small radiation doses the deeper traps are filled preferentially but at higher doses the glow curve is domi-

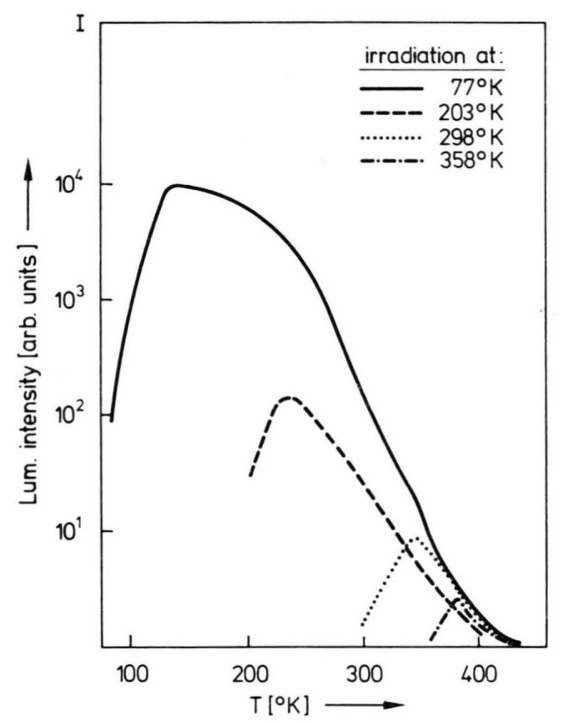

Fig. 3. TL Glow curves of silica gel outgassed at $800^{\circ} \mathrm{K}$, $\gamma$-irradiated to $3.8 \mathrm{Mrad}$ at the temperatures indicated in the fig. and heated at a rate of $5^{\circ}$ per min.

nated by the more numerous shallow traps. The increase of the trap concentration towards the band edge is also demonstrated in Figure 3. Different samples were irradiated to identical doses but at different temperatures. The intensity increases steeply as the temperature of irradiation is lowered while the high temperature tail of the curve changes slightly. In Figures 1 and 2 the intensity increases with adsorbed dose. The integrated glow curve, i. e. the total light sum emitted, as a function of radiation dose is plotted in Figure 4. It shows a linear growth up to about $1 \mathrm{Mrad}$ with practically no further increase for higher doses. It should be mentioned that this behaviour is in sharp contrast to the growth of the center concentration as observed by optical

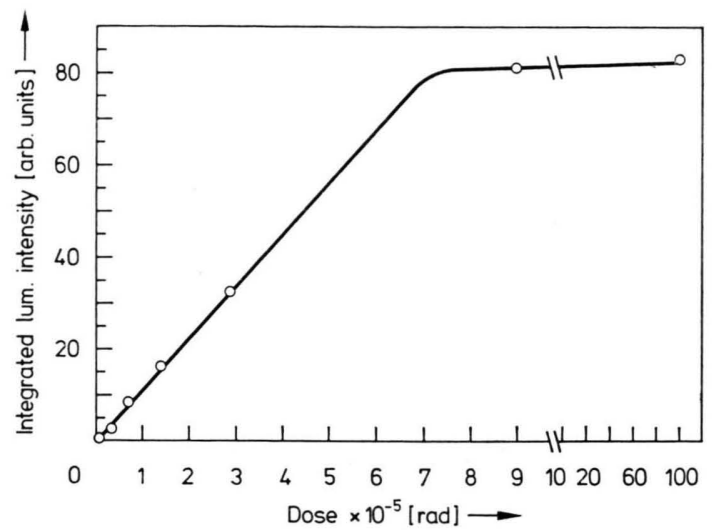

Fig. 4. Integrated TL glow curves, i. e. amount of light emitted within the glow curve, as a function of radiation dose. Samples were outgassed at $800^{\circ} \mathrm{K}, \gamma$-irradiated at $77^{\circ} \mathrm{K}$ and heated at a rate of $5^{\circ}$ per min.

absorption ${ }^{15}$, esr ${ }^{16}$ or chemical determination ${ }^{17}$ found to continue far beyond a dose of 1 Mrad. As may be judged from Figures 1 and 2, the glow curves are composed of several unresolved glow peaks. Thermal cleaning has been used in an attempt to better resolve the glow curves. Several samples irradiated at $77^{\circ} \mathrm{K}$ where heated to some temperature within the glow curve and the luminescence allowed to decay at that temperature. Subsequent cooling to $77^{\circ}$ and linear heating resulted in the glow curves shown in Figure 5. After thermal

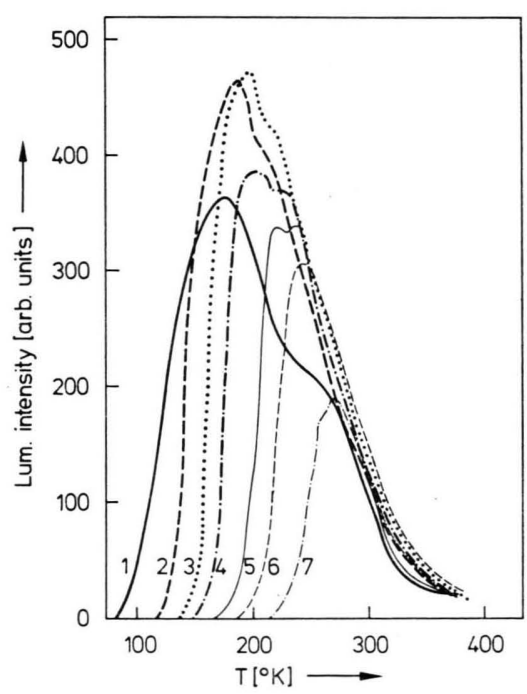

Fig. 5. TL glow curves after thermal cleaning. Silica gel outgassed at $773^{\circ} \mathrm{K}, \gamma$-irradiated at $77^{\circ} \mathrm{K}$ to $7.15 \times 10^{4} \mathrm{rad}$, thermal cleaning at 1 ) without cleaning; 2) $119^{\circ} \mathrm{K}$; 3) $141^{\circ} \mathrm{K}$; 4) $155^{\circ} \mathrm{K}$; 5) $181^{\circ} \mathrm{K}$; 6) $198^{\circ} \mathrm{K}$; 7) $224^{\circ} \mathrm{K}$; heating rate $5^{\circ}$ per min. 
cleaning at temperatures up to $155^{\circ} \mathrm{K}$, the intensity has increased indicating that the occupancy of certain traps has changed. A reduction of the number of charges in shallow traps is accompanied by an increase of charges in somewhat deeper traps, whereas the still deeper traps determining the high temperature wing of the glow curves are not affected. Thermal cleaning at $119^{\circ} \mathrm{K}$ leads to an even larger amount of emitted light than observed prior to cleaning. It thus appears that the quantum efficiency has become larger.

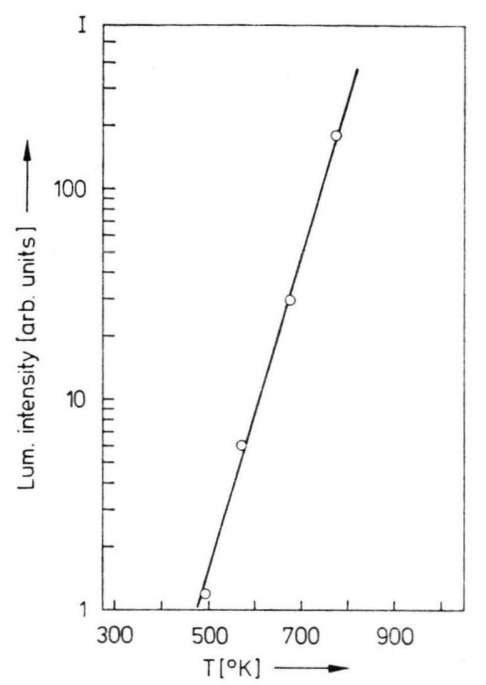

Fig. 6. TL intensity in the glow curve maxima as a function of the temperature at which silica gel was outgassed prior to $\gamma$-irradiation to $2.66 \times 10^{5} \mathrm{rad}$. Heating rate $10^{\circ}$ per min.

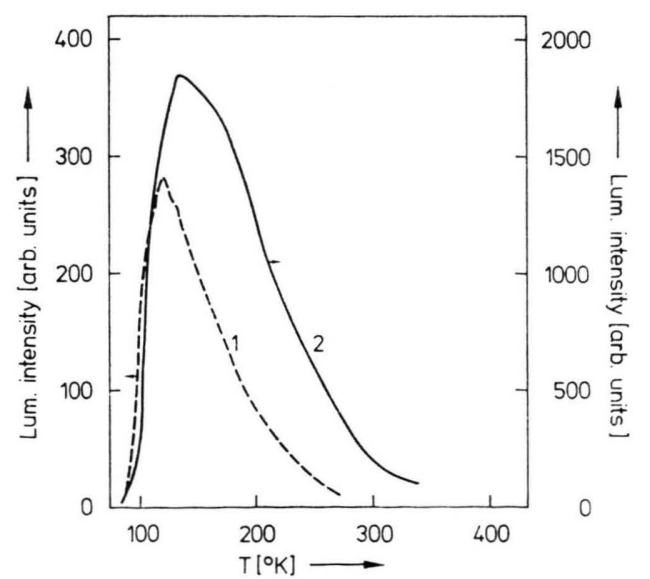

Fig. 7. TL glow curves of silica gel outgassed at 1) $663^{\circ} \mathrm{K}$; 2) $773^{\circ} \mathrm{K}$; and $\gamma$-irradiated to $2.66 \times 10^{5} \mathrm{rad}$. Heating rate $10^{\circ}$ per min.

\subsection{Influence of Surface Treatment}

A change of the outgassing temperature of the sample causes a drastic change in the intensity and a shift of the maximum of the glow curve. The intensity as a function of the outgassing temperature is shown in Figure 6. Within the temperature range investigated the intensity doubles when the temperature is increased by $40^{\circ}$. Figure 7 shows the glow curves for samples outgassed at 663 and $773{ }^{\circ} \mathrm{K}$, respectively. In addition to the intensity increase

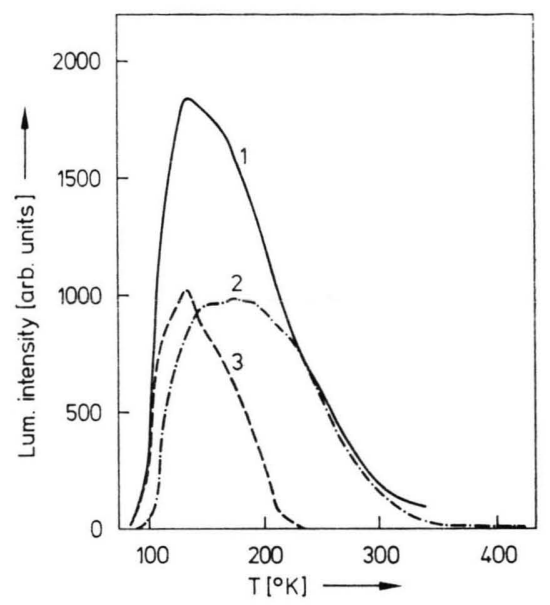

Fig. 8. TL glow curves of silica gel. 1) outgassed at $773^{\circ} \mathrm{K}$; 2) heated to $1273^{\circ} \mathrm{K}$ in the presence of air and outgassed at $773{ }^{\circ} \mathrm{K} ; 3$ ) difference between glow curves 1 and 2. Radiation dose $2.66 \times 10^{5} \mathrm{rad}$; heating rate $10^{\circ}$ per $\min$.

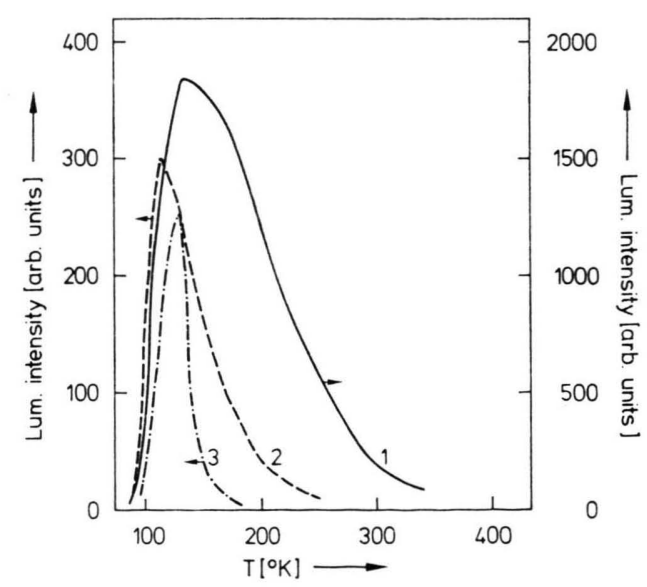

Fig. 9. TL glow curve of silica gel as affected by adsorbed hydrogen gas. 1) without hydrogen; 2) hydrogen absorbed prior to irradiation; 3) hydrogen adsorbed subsequent to irradiation. Outgassed at $773^{\circ} \mathrm{K}, \gamma$-irradiated to $2.66 \times 10^{5}$ rad; heating rate $10^{\circ}$ per min. 
the maximum is shifted by $14^{\circ}$ to a higher temperature for the $773^{\circ} \mathrm{K}$ treated sample. The shift is due to the high temperature wing of the glow curve being comparatively more enhanced than the low temperature wing. It may then be concluded that the thermal treatment favors the formation of deeper traps. Such a conclusion is supported by an experiment in which one sample first was heated to $1273{ }^{\circ} \mathrm{K}$ in the presence of air and then cooled to room temperature before it was outgassed and irradiated identically to a second sample. Comparison of glow curves 1 and 2 in Fig. 8 shows a loss of intensity (curve 3 ) as a result of the $1275^{\circ} \mathrm{K}$ treatment. It is of interest to note that this thermal treatment has considerably reduced the specific surface area of the gel. It thus appears that the loss of intensity occurring almost exclusively in the low temperature wing of the glow curve, i. e. at the expense of shallow traps, is somehow related to the loss of surface area.

In the experiments described thus far all samples were irradiated and the luminescence measured while kept in vacuum $\left(\sim 10^{-5}\right.$ Torr $)$. Except for chemisorbed water affected by the temperature of outgassing, surface effects caused by adsorbed gases are thus excluded. Significant differences of TL are observed with samples either irradiated in the presence of gases or to which gases were added subsequent to irradiation. As an example, the effect of hydrogen gas is shown in Figure 9. Adsorption of hydrogen both prior and subsequent to irradiation reduces the TL glow curve intensity to a few percent of that of a sample kept in vacuum. The similarity of the glow curves obtained with the hydrogen treated samples and the one obtained with a sample kept in vacuum but outgassed at a $110^{\circ}$ lower temperature (Fig. 7, curve 1) is striking. It thus appears that the effect caused by an increase of the outgassing temperature is reversed by the presence of hydrogen. The post irradiation adsorption of hydrogen demonstrates an effect on trapped charges. Experiments are described in II, which concern the interaction of trapped charges with molecules in the act of adsorption. It will be shown there that the interaction leads to what is called radiosorptionluminescence.

Similar to the action of hydrogen, TL is strongly reduced when samples are treated with oxygen or nitrous oxide, whereas nitrogen and helium have practically no effect. In samples of low specific surface area TL was not affected to a measurable extent by any of the gases mentioned suggesting that the action of adsorbed molecules is confined to a certain range penetrating the surface.

\subsection{Decay Kinetics of Luminescence}

Samples irradiated at $77^{\circ} \mathrm{K}$ do not luminescence if kept in the dark. When the temperature is raised to some temperature within the glow curve, decay of luminescence is observed. The luminescence decay obeys the hyperbolic relation $I \propto I_{0}(1+k t)^{-\beta}$ as is seen from Fig. 10 where a plot of log intensity $I$ versus log decay time $t$ yields straight lines.

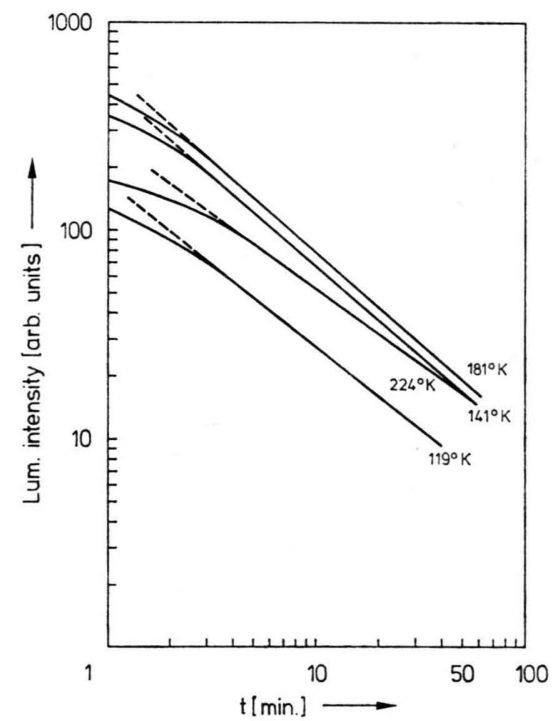

Fig. 10. Isothermal decay of luminescence in silica gel at various temperatures. Logarithmic plot of luminescence intensity $I$ versus decay time $t$.

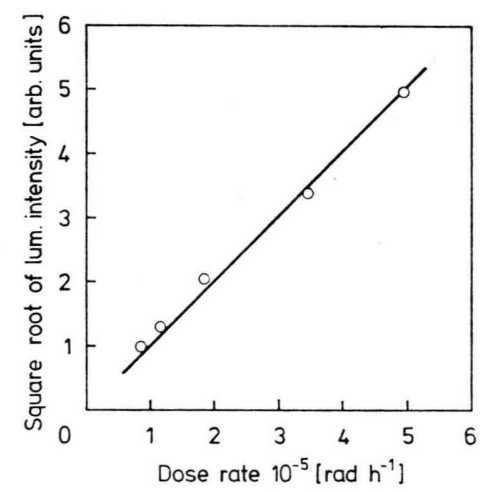

Fig. 11. Plot of the square root of luminescence intensity versus dose rate of $\gamma$-irradiation. Silica gel was irradiated at ambient temperature for $2 \mathrm{sec}$ (from K. Breitkreutz, reference ${ }^{15}$ ). 
For very small radiation doses the initial luminescence intensity depends strongly on the dose rate ${ }^{15}$. This is demonstrated with samples irradiated at room temperature to doses of the order of a hundred rads at various dose rates and with the luminescence intensity being measured a constant time after the irradiation was interrupted. The result is shown in Fig. 11 where a plot of the square root of the luminescence intensity versus the dose rate is seen to yield a straight line. It should be mentioned that the irradiation dose applied is several orders of magnitude smaller than the megarad dose needed to obtain the maximum luminescence, i. e. the number of luminescent centers excited is only a small fraction of the total number of luminescent centers.

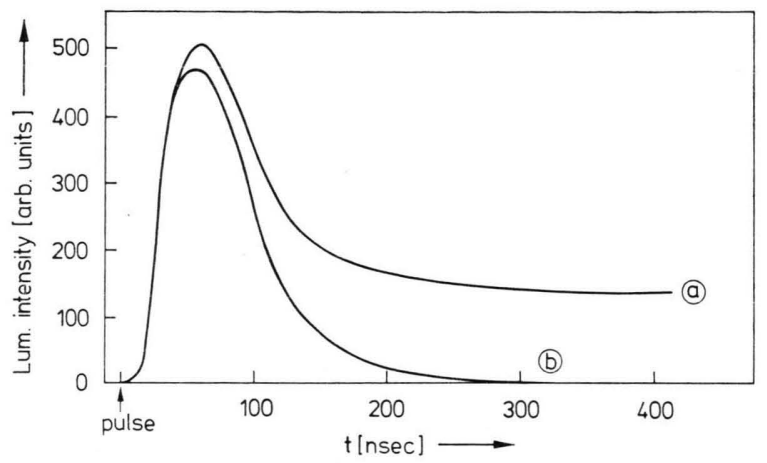

Fig. 12. Photo stimulated luminescence in $\gamma$-irradiated silica gel at $77^{\circ} \mathrm{K}$. Curve a - Observed with a $\gamma$-irradiated sample. Inserting a filter cutting off $\lambda<700 \mathrm{~nm}$ leads to curve b. Curve b - Pulse of GaAs-laser light $(905 \mathrm{~nm})$ observed with a sample prior to $\gamma$-irradiation.

Luminescence is also stimulated by incident light. An experiment was performed in which samples irradiated and kept at $77^{\circ} \mathrm{K}$ were exposed to a light flash of $905 \mathrm{~nm}$ wavelength emitted by a GaAs-laser. The photomultiplier output was recorded as oscilloscopic traces at various time scales. From several such traces Fig. 12 was constructed. It shows in the lower curve the intensity recorded after the light has passed a filter cutting off wavelengths $<700 \mathrm{~nm}$. An identical curve was obtained with unirradiated samples and without a filter. It may be concluded, therefore, that the curve is due to the laser light. The upper curve was obtained from irradiated samples but without use of a filter. The difference of the two curves thus is the light of wavelength shorter than $700 \mathrm{~nm}$ emitted from the samples on stimulation with $905 \mathrm{~nm}$ light. It should be noted that the inten- sity of the emitted light rises slower to its maximum than the intensity of the stimulating light; furthermore, the emission continues long after the stimulation has ceased.

In fact luminescence was still measurable 300 microseconds after the $0.2-0.3$ microsecond laser pulse. The stimulated emission decays in its later stage in a hyperbolic fashion similar to the decay shown in Figure 10.

\subsection{Emission Spectrum}

The spectral distribution has one maximum at $450 \mathrm{~nm}$. The width of the band at half of the peak height is roughly $0.8 \mathrm{eV}$. The short wavelength side extends into the region below $400 \mathrm{~nm}$ which is outside the $400-700 \mathrm{~nm}$ range covered by the filter used. Although resolution of the filter is poor, obviously the same transition occurs in all observed RTL.

\section{Discussion}

For a solid of $450 \mathrm{~m}^{2}$ per gram surface area and a density around 2.5 the size of an idealized globular particle assuming no pores would be of the order of $60 \AA$ in diameter, putting the volume to surface ratio of its constituents to around 4 . The bulk material of silica gel consists of $\mathrm{SiO}_{4}$-tetrahedra with the $\mathrm{Si}-\mathrm{O}-\mathrm{Si}$ bond angle randomly arranged. At the surface a large number of silanol groups $(\mathrm{Si}-\mathrm{O}-\mathrm{H})$ rather than siloxane groups $(\mathrm{Si}-\mathrm{O}-\mathrm{Si})$ exists.

The luminescent results may be discussed in terms of the band model modified by taking into account the disordered structure typical for liquids and glasses ${ }^{18}$. The main feature is the introduction of localized levels originating from the lack of periodicity which is due to the randomness of bond angles. Assuming that short range order is preserved, there are in addition the levels originating from specific defects such as vacancies and other perturbations known from the crystalline state.

\subsection{Trap Levels and Retrapping}

The observed dependence of the glow curves on the temperature of irradiation (Fig. 3) suggests several trapping levels distributed in energy and an increase of level density towards the band edge. From the dependence of the glow curve structure on the radiation dose (Figs. 1 and 2) it may be concluded that the different trap levels are not filled 
with identical rates. It further implies that the glow curves are composed of several groups of levels having certain activation energies rather than consisting of levels with a continuum of energy values. The redistribution of trapped charge carriers observed on thermal cleaning (Fig. 5) supports this notion. It also provides evidence for retrapping. Based on the occurence of peaks and shoulders in the glow curves, a geometrical analysis allows an estimate of the activation energies involved in the release of charges from traps. Several methods have been applied and it seems safe to state that most of the emitted light $(\sim 90 \%)$ is due to traps of $0.1-$ $0.2 \mathrm{eV}$ depth. The remaining luminescence involves charge release from traps up to $0.7 \mathrm{eV}$ depth. In principle, similar results were previously obtained with silicate glass ${ }^{19-21}$, fused quartz ${ }^{19}$ and vitreous $\mathrm{GeO}_{2}{ }^{22}$ and interpreted to reflect a feature of the glassy state.

\subsection{Relation of Luminescence to Color and Paramagnetic Centers}

There is no simple (if any) relation between the formation under irradiation of color-, paramagneticor chemically active centers on one side and the occurrence of luminescence on the other side. The former were found still to increase in concentration after absorption of $70 \mathrm{Mrad}$ whereas luminescence is saturated near $1 \mathrm{Mrad}$ (Figure 4). In contrast to luminescence the esr signal of a sample irradiated a $77^{\circ} \mathrm{K}$ changes only slightly when warmed to room temperature between two measurements at $77^{\circ} \mathrm{K}$. Finally, there is no luminescence observable above $400{ }^{\circ} \mathrm{K}$ up to $600^{\circ} \mathrm{K}$, whereas the bulk of color and paramagnetic centers disappear in that temperature region.

It is tentatively suggested that the shallow traps responsible for the luminescence are due to the randomness of the $\mathrm{Si}-\mathrm{O}-\mathrm{Si}$ bond angle, the characteristic feature of the glassy state. In contrast it appears that color- and paramagnetic centers are associated with specific defects (deep traps $>0.7 \mathrm{eV}$ ) such as vacancies and impurities common in both the glassy and crystalline state of silica ${ }^{23-25}$.

\subsection{Kinetics}

Since luminescence is presumed to result from a bimolecular process - excitation of a luminescent center originating from the recombination of charges of opposite sign - the decay of luminescence may be expected to obey second-order kinetics. The observed decay fits the broken hyperbola $I \propto I_{0} /$ $(1+k t)^{\beta}$ where $\beta<2$ (Figure 10). This relation which assumes second order kinetics to be applicable, was derived by Adirovich ${ }^{26}$ as an approximation for the main region of the decay curve in the case of saturated luminescence and rate constants for retrapping larger than for recombination (see also reference ${ }^{27}$ ). The indication of strong retrapping thus obtained is supported by the results on thermal cleaning mentioned above and by the fact that a long lasting luminescence is observed in the laser pulse experiment (Figure 12).

The observed linear dependence of the square root of the initial luminescence intensity on the dose rate $(V I \propto i$; Fig. 11$)$ is consistent with a bimolecular mechanism of luminescence. AntonowRomanovski ${ }^{28}$ has considered the kinetics of electron transitions for a bimolecular mechanism which is given by

$$
\mathrm{d} n / \mathrm{d} \vartheta=\varkappa i\left(n_{1}-n\right)-k n^{2}
$$

with $n$ the number of excited luminescence centers, $n_{1}$ the total number of luminescence centers, $\varkappa$ and $k$ the probabilities for excitation of a center and the emission of an excited center, respectively, and $i$ the intensity of the exciting radiation.

At sufficiently small excitation, i.e. excitation for such a short period of time that $n \ll n_{1}$, Eq. (1) then reduces to

$$
\mathrm{d} n / \mathrm{d} \vartheta \approx \varkappa i n_{1} .
$$

At the moment the excitation ceases, the number of excited luminescence centers $n$ will be

$$
n \approx \varkappa n_{1} \Theta i
$$

where $\Theta$ is the time the phosphor was exposed to exciting radiation. The initial intensity in the case of a bimolecular mechanism is then given by

or

$$
I=k n^{2}=k\left(\varkappa n_{1} \Theta\right)^{2} i^{2}
$$$$
I \propto i^{2} \text {. }
$$

In the simple bimolecular scheme [Eq. (1)], retrapping and the possibility of a simultaneous occurrence of monomolecular and bimolecular processes have not been taken into account. This does not, however, change the principal result that $I$ is a quadratic function of $i$ [Eq. (4)] as discussed by Adirowich ${ }^{26}$.

\subsection{Surface Effects}

The main effect of increasing the degassing tem- 
perature of a silica gel is to replace the large number of silanol groups at the surface by siloxane groups ${ }^{29,30}$. It is suggested that the removal of hydroxyl groups is responsible for the increase of the luminescence observed at the higher degassing temperatures (Figure 6). The effect of hydroxyl groups as well as the suppression of luminescence seen with adsorbed oxygen and hydrogen (Fig. 9) may be accounted for by assuming that they form impurity levels competing with other levels located within the band gap.

These impurity levels open a channel for the release of charges or their precursors which otherwise would become stabilised in a way that enables luminescence. In terms of chemical reactions it has been discussed previously that electron trapping occurs by the formation of hydrogen atoms from hydroxyl groups ${ }^{31}$. Electron trapping occurs also at adsorbed oxygen resulting in the formation of $\mathrm{O}_{2}{ }^{-}$ anion radicals $8,25,32$. The action of hydrogen seems to be largely on holes. Trapped holes are not formed when hydrogen is present during irradiation and once being formed are easily annihilated on adsorption of hydrogen ${ }^{7,32-36}$. The adsorption, found to be irreversible even at $790{ }^{\circ} \mathrm{K}^{17}$, may result from a reaction between the hole trapped on oxygen and hydrogen to reform hydroxyl groups

$$
\left(\equiv \mathrm{Si}-\mathrm{O}^{*}+\mathrm{H}^{*} \rightarrow \equiv \mathrm{Si}-\mathrm{OH}\right)^{33,35} \text {. }
$$

In fact TL observed with a silica gel of low $\mathrm{OH}$ concentration but irradiated in the presence of hydrogen is strikingly similar to the TL observed with a gel of higher $\mathrm{OH}$ concentration (Figures 9 and 7). Not only has the presence of hydrogen suppressed the luminescence to a value typical for a gel of higher $\mathrm{OH}$ content but it has also shifted the intensity maximum to the lower temperature of such

$1 \mathrm{H}$. W. Kohn, in: Actions Chimiques et Biologiques des Radiation, Masson, Paris 1967.

2 E. H. Taylor, Adv. Catalysis 18, 111 [1968].

3 G. M. Zhabrova, V. I. Vladimirova, B. M. Kadenatsi, V. B. Kazanskii, and G. B. Pariiskii, J. Catalysis 6, 411 [1966].

4 J. G. Rabe, B. Rabe, and A. O. Allen, J. Phys. Chem. 70, 1098 [1966].

5 O. Edlund, P. O. Kinell, A. Lund, and A. Shimizu, J. Chem. Phys. 46, 3679 [1967].

6 P. K. Wong, and J. E. Willard, J. Phys. Chem. 72, 2623 [1968].

7 R. R. Hentz and D. K. Wickenden, J. Phys. Chem. 73, 817 [1969].

8 Y. Yamada, A. Hasegawa, and M. Miura, Bull. chem. Soc. Japan 42, 1836 [1969].

9 P. K. Wong and A. O. Allen, J. Phys. Chem. 74, 774 [1970]. a gel. Since Kazansky et al. ${ }^{31}$ observed that hydrogen atoms originating from the radiolysis of hygroxyl groups at $77^{\circ} \mathrm{K}$ become mobile at $125-$ $150{ }^{\circ} \mathrm{K}$, it is proposed that TL may be affected in that temperature region. The energy liberated on the combination of hydrogen atoms or on reaction of hydrogen atoms with holes trapped at nonbridging oxygen as well as from any other exothermic process could lead to a release of charges from traps.

It will be shown in Part II that such processes indeed can empty traps. Another piece of evidence is obtained from the result of Figure 8 . The loss of luminescence (curve 3 ) observed with the $1275^{\circ} \mathrm{K}$ treated gel as compared to a gel not subjected to such treatment coincidentally occurs in that temperature region $\left(120-150^{\circ} \mathrm{K}\right)$ in which mobile hydrogen atoms would be expected to affect the RTL. Since the thermal treatment strongly decreases the $\mathrm{OH}$ concentration with a concommitant reduction of surface area, there is no longer a possibility of interference of surface reactions. This is confirmed by the observed failure of adsorbed gases to affect TL in the low surface area gel. It indicates a limited range of action of adsorbed molecules which, however, it not less than $30 \AA$, taking into account the particle size of high surface area gel as discussed in the beginning and the almost complete suppression of TL.

\section{Acknowledgement}

Financial support from the Deutsche Forschungsgemeinschaft is greatefully acknowledged. Thanks are due M. Wilhelm for his advice on the laser experiment and Dr. Breitkreutz who contributed the results shown in Figure 11.

10 G. K. Boreskow, V. B. Kazanskii, Yu. A. Mishchenko, and G. B. Pariiskii, Dokl. Akad. Nauk. SSSR 157, 384 [1964].

11 R. R. Hentz, L. M. Perkey, and R. H. Williams, J. Phys. Chem. 70, 731 [1966].

12 J. G. Rabe and G. Joppien, Z. Naturforsch. 24 a, 796 [1969].

13 D. I. Baasow, J. A. Kolbanovsky, and L. S. Polak, Int. J. Radiat. Phys. Chem. 1, 53 [1969].

14 P. Kirmaier, Ph. D. Thesis, Techn. Universität Berlin (1974).

15 K. Breitkreutz, Ph. D. Thesis, Techn. Universität Berlin (1970).

16 I. Kalinowski, P. Kirmaier, and J. G. Rabe, unpublished results.

17 J. G. Rabe, Int. J. Radiat. Phys. Chem. 5, 301 [1973].

18 H. Fröhlich, Proc. Roy. Soc. London A 188, 521 [1947].

19 T. Kikuchi, J. Phys. Soc. Japan 13, 526 [1958]. 
20 C. Bettinali and G. Ferraresso, J. Chem. Phys. 44, 2262 [1966].

21 S. A. A. Winer and R. Chen, J. Chem. Phys. 51, 4530 [1969].

22 H. Böhm, Phys. Chem. Glasses 11, 177 [1970].

23 E. Lell, N. Kreidl, and J. R. Hensler, Radiation Effects in Quartz, Silica and Glasses, "Progress in Ceramic Science", Vol. 4, Pergamon Press, London 1966.

24 G. M. Muha, J. Phys. Chem. 70, 1390 [1966].

25 P. K. Wong and J. E. Willard, J. Phys. Chem. 73, 2226 [1969].

26 E. I. Adirowitsch, Einige Fragen zur Theorie der Lumineszenz der Kristalle, Akademie-Verlag, Berlin 1953.

27 J. G. Rabe, Int. J. Radiat. Phys. Chem. 6, 275 [1974].

28 W. W. Antonow-Romanowski, Ber. d. Akad. d. Wiss. d. UdSSR 2, 93 [1936], and quoted in Ref. 26.
29 J. B. Peri and A. L. Hensley, jr., J. Phys. Chem. 72, 2926 [1968].

30 C. G. Armistead, A. J. Tyler, F. H. Hambleton, S. A. Mitchell, and J. A. Hockey, J. Phys. Chem. 73, 3947 [1969].

31 V. B. Kazansky, G. B. Pariisky, and V. V. Voevodsky, Disc. Faraday Soc. 31, 203 [1961].

32 J. G. Rabe and K. Breitkreutz, Ber. Bunsenges. phys. Chem. 75, 1123 [1971].

33 G. B. Pariisky, Yu. A. Mishchenko, and V. B. Kazansky, Kinetika i Kataliz 6, 625 [1965].

34 H. W. Kohn, J. Catalysis 2, 208 [1963].

35 G. M. Muha and D. J. C. Yates, J. Phys. Chem. 70, 1399 [1966].

36 S. A. Surin, B. N. Shelimov, and V. B. Kazanskii, Khimiya Vysokikh Energii 5, 443 [1971]. 